\title{
The Structure of Organizational Behavior Management: Identification, Selection and Feedback
}

\author{
Jie ZHEN ${ }^{\star}$ \\ East China University of Political Science and Law, Shanghai, China \\ jetzhen@163.com
}

Keywords: Organizational behavior management, Identification, Selection, Feedback.

\begin{abstract}
The structure of organizational behavior management is composed of identification, selection and feedback. The fundamental content of identification includes clarifying the types and forms of various organization behavior, justifying the feature and function of concrete action, grasping the development conditions of organization conduct. The selection of organizational behavior must first be based on two basic essentials, one is the strategic goal of the organization; the other is the external production environment. Organizational behavior feedback refers to the evaluation of its performance under certain circumstances.
\end{abstract}

\section{Introduction}

Organizational behavior focuses on the action rules involving the individual, group and organization and the interaction relationship among the three levels, so as to improve the capacity of managers to identify, select and optimize the employees' conduct and enhance the efficiency of the organization operation. Prior to the optimization of behavior, the behavior should be evaluated first, and the two aspects constitute the content of behavioral feedback. Therefore, this paper proposes and constructs the "three dimensions" structure of organizational behavior which includes identification, selection and feedback. Among them, behavior identification is the analysis of the state of things and the reasons for its appearance before the event; the behavior selection is to match the environment, task and behavior during the event from the perspective of self-discipline and heteronomy; the behavior feedback is an effect evaluation and processing after the event. The three stages form the structural content of behavior management, which aims to improve and optimize organizational efficiency.

\section{Identification of Organization Behavior}

The identification of organizational behavior is the analysis of the state and manifestation of the organization's internal and external behavior. Its fundamental content includes three aspects which are clarifying the types and forms of various organization behavior, justifying the feature and function of concrete action, grasping the development conditions of organization conduct. Through identification, the organization can prepare for the selection of its behavior.

Take the business process as an example, the identification instrument is represented by Process and Enterprise Maturity Model (PEMM) constructed by Michael Hammer (2004). [1] This model focuses on the behavior of the individual, group and organization inside the enterprise, and describes the behavioral features of each level and then analyzes the corresponding function. In the context of the growing inter-organizational behavior relationship, PEMM also attaches great importance to the behavioral patterns of various aspects in the process of facing external customers. Meanwhile, PEMM identifies business process from five aspects involving design, performer, leader, infrastructure, metrics which become process enablers. By grasping these factors, we can clarify the behaviors' production conditions and the long-term operation effect of the process.

In general, the recognition of the organizational behavior reflected by PEMM (which can evaluate any industry process) [2] extends from the internal organization behavior to the external organization behavior at the higher levels. Among them, each of the enablers is interdependent and indispensable. For example, the process leader cannot implement a good process design if the leader 
is too weak; the performer will be unable to complete the execution of the process design if he or she lacks the training. The strength of process enablers is divided into four levels: P-1, P-2, P-3 and P-4 which are progressive increment (see table 1, the specific contents of the indicators omitted). For the performers, the P-1 staff only know the existence of the process and its measurable indicators; the P-2 staff must describe the whole process and its current link of the process; the P-3 staff should explain how their work will affect the performance of the enterprise; the employees at the P-4 level must know how their work affects their customers and suppliers, which is to know more about inter-organizational behavior.

Table 1. Identification of Business Process Maturity

\begin{tabular}{|c|c|c|c|c|c|}
\hline Enabler & Variable & $\mathrm{P}-1$ & $\mathrm{P}-2$ & $\mathrm{P}-3$ & $\mathrm{P}-4$ \\
\hline \multirow{3}{*}{ Design } & Objective & & & & \\
\hline & Inter-Processes Relations & $\ldots \ldots$ & $\ldots \ldots$ & $\ldots \ldots$ & $\ldots \ldots$ \\
\hline & Record & $\ldots \ldots$ & $\ldots \ldots$ & $\ldots \ldots$ & $\ldots \ldots$ \\
\hline \multirow{3}{*}{ Performer } & Knowledge & & & & \\
\hline & Skill & $\ldots \ldots$ & $\ldots \ldots$ & $\ldots \ldots$ & $\ldots \ldots$ \\
\hline & Behavior & $\ldots \ldots$ & $\ldots \ldots$ & $\ldots \ldots$ & $\ldots \ldots$ \\
\hline \multirow{3}{*}{ Leader } & Rank & $\cdots \cdots$ & $\cdots \cdots$ & $\cdots \cdots$ & $\cdots \cdots$ \\
\hline & Activity & & & & \\
\hline & Right & $\ldots \ldots$ & $\ldots \ldots$ & $\ldots \ldots$ & $\ldots \ldots$ \\
\hline \multirow{2}{*}{ Infrastructure } & Information System & & & & \\
\hline & Human Resource & $\ldots \ldots$ & ....... & $\ldots \ldots$ & $\ldots \ldots$ \\
\hline \multirow{2}{*}{ Metrics } & Definition & & & & \\
\hline & Application & $\ldots \ldots$ & ....... & $\ldots \ldots$ & $\ldots \ldots$ \\
\hline
\end{tabular}

When identifying the process maturity determined by the strength of the enablers, the process itself is at p- 1 level if the five factors are all at p- 1 level, and so on. The process will still be at a lower level if there is one factor staying at the level while the other four factors reaching a higher level. In particular, if one enable is so weak that it even cannot reach at P-1 level, then the process default to the level of P-0, namely the natural state when the enterprise has not yet committed to the development of the business process. Specifically, the p-0 level process cannot maintain in a stable state; the p-1 level process is reliable and stable; the p-2 level process can achieve excellent performance; the p-3 level process can acquire optimal results; the p-4 level process is the best one which extends beyond the enterprise boundaries to upstream suppliers and downstream customers.

\section{Selection of Organization Behavior}

In general, alternative organizational behavior is more than one type after going through the identification stage of organizational behavior. Because organizational behavior selection is the key to the survival and development of the organization, the organization needs to match the environment, task and organizational behavior based on the perspective of self-discipline and heteronomy. The selection of organizational behavior must first be based on two basic essentials, one is the strategic goal of the organization; the other is the external production environment. Then, the organization needs to consider the method of behavioral choice, whether it is democratic negotiation or personal decision, and whether it is dependent on intuition or rigorous analysis, which are the premise of making a choice. In addition, the possible costs, benefits and degree of risk during the behavioral choice are the involved content in the selection process.

We still take the process change in organizational behavior as an example to match the organizational capability and process. Specifically, the supporting environment of the enterprise 
includes four organizational capabilities, namely leadership, culture, expertise and governance. The enterprise must first have all these abilities, and then it is possible to implement the requirements of the process enablers and maintain the long-term performance of the process. In other words, the enterprise capability must reach the basic level to initiate the process change project. Meanwhile, the enterprise must also reach a higher level of competence to achieve greater progress. The enterprise capability is divided into four levels: E-1, E-2, E-3 and E-4. The maturity of the enterprise is at the first level if an enterprise is at E-1 level, and so on.

Different levels of each capacity have corresponding specific definition (see table 2, the contents of the indicators omitted). For example, if the organizational culture is at the E- 1 level, it means that it has a certain team cooperative experience. But to go further, companies must have the E-2 culture, which means that companies often need to organize cross-functional project teams and familiarize employees with teamwork. To reach the E-3 level, teamwork must be the standard way of working. When an enterprise is used to working with suppliers and customers, it will rise to the E-4 level.

In fact, this kind of classification also provides a selection method for the matching of organizational capability and process change. If the enterprise needs to choose a more mature business process, it must have a higher level of organizational capability, which would require the enterprise improve the corresponding organization behavior in accordance with the requirements described in table 2 and match the business process level in leadership, culture, professional skills and governance.

Table 2 Identification of Business Process Maturity

\begin{tabular}{|c|c|c|c|c|c|}
\hline Capability & Variable & E-1 & E-2 & E-3 & E-4 \\
\hline \multirow{4}{*}{ Leadership } & Comprehend & & & & \\
\hline & Coordination & $\cdots \cdots$ & $\ldots \ldots$ & $\ldots \ldots$ & $\ldots \ldots$ \\
\hline & Behavior & $\ldots \ldots$ & $\ldots \ldots$ & $\ldots \ldots$ & ....... \\
\hline & Style & & & & \\
\hline \multirow{4}{*}{ Culture } & Teamwork & & & & \\
\hline & Customer Orientation & $\cdots \cdots$ & $\ldots \ldots$ & $\ldots \ldots$ & $\ldots \ldots$ \\
\hline & Duty & $\ldots \ldots$ & $\ldots \ldots$ & $\ldots \ldots$ & $\ldots \ldots$ \\
\hline & Attitude to Change & & & & \\
\hline \multirow{2}{*}{$\begin{array}{l}\text { Professional } \\
\text { Skill }\end{array}$} & Employee & $\cdots \cdots$ & $\cdots \cdots$ & $\cdots \cdots$ & $\cdots \cdots$ \\
\hline & Method & & & & \\
\hline \multirow{3}{*}{ Governance } & Process Model & & & & \\
\hline & Responsibility & $\ldots \ldots$ & $\ldots \ldots$ & $\ldots \ldots$ & $\ldots \ldots$ \\
\hline & Integration & & & & \\
\hline
\end{tabular}

\section{Feedback of Organization Behavior}

Organizational behavior feedback refers to the evaluation of its performance under certain circumstances. If the performance is satisfied, the current status is maintained. If the performance needs to be improved, some measures should be taken to optimize organizational behavior and ultimately enhance the efficiency of the organization.

As previously mentioned, process enablers and enterprise competence constitute a complete framework, which can be applied by the companies to evaluate the maturity of the business process and the acceptability of process innovation, and then the organizational behavior can be optimized by the companies.

In order to further improve its performance, the company decides to elevate the process enablers from P-2 to P-3 level and guide the leadership team to use the PEMM framework to find the areas 
for improvement. In this process, the company's senior managers and front-line employees clarify their differences and have conducted sufficient communication to make the assessment result accurate. When organizational capability matches the corresponding level of process change and achieves high performance, maintaining the high performance of the process becomes the key to further focus on organizational behavior.

\section{Acknowledgement}

This research was financially supported by East China University of Political Science and Law under Grant 2017 Science Research Project, Shanghai Social Sciences Planning Project under Grant 2015JG009-BGL307 and the National Social Science Foundation of China under Grant 17BGL099.

\section{References}

[1] Michael Hammer. A New Tool for Process Reengineering: PEMM Framework. Harvard Business Review, 2007(10):141-154.

[2]LIN Yongyi, LI Minqiang. Study on the Maturity Model of Enterprise Business Process. Modern Management Science. 2008(7):93-95. 